\title{
Validation of the use of an automatic diluter in HPLC analyses by comparison with traditional methods
}

\author{
J. Vial ${ }^{1}$, A. Jardy ${ }^{1, *}$, P. Anger ${ }^{2}$ and G. Gorge ${ }^{2}$ \\ ${ }^{1}$ Laboratoire " environnement et chimie analytique », associé au CNRS, École Supérieure de Physique \\ et Chimie Industrielles de la Ville de Paris, 10 rue Vauquelin 75005 Paris, France \\ ${ }^{2}$ Centre de Recherche de Vitry Alfortville, Rhône-Poulenc Rorer, \\ 13 Quai Jules Guesde, 94400 Vitry sur Seine, France
}

\begin{abstract}
In an analysis protocol, dilution is nowadays the last step that is never (or seldom) fully automated. Yet special appliances called diluters are commercially available. In spite of the potential advantages in time and solvent saving, analysts are still reluctant to use these diluters, mainly because of the insufficient proof that they are able to give accurate and precise results. Validation by gravimetry did not represent an irrevocable argument since it could not account for memory effect. That is the reason why we chose to carry out our validation of the dilution step in HPLC analyses with an automatic diluter through a comparison with classical methods using flasks and pipettes or burettes. A parent solution made of concentrated Spiramycin was diluted by the various methods. The control analysis was operated using HPLC with UV detection. The experimental design involved two operators and results obtained were processed using statistical tools such as ANOVA and regression. Conclusions were that in any case the diluter was at least as effective as traditional methods. Observed variability introduced by dilution was $0.30 \%$ RSD with diluter, and $0.45 \%$ RSD with classical methods. In addition, as was shown by the results of the lack of fit test, diluter accuracy is quite compatible with linearity studies in HPLC. Consequently, it is possible and even advantageous to use a diluter instead of manual methods.
\end{abstract}

Key words. Dilution error - automatic diluter - validation - ANOVA - HPLC.

\section{Introduction}

Today, analytical methods tend to be fully automated since this improves the repeatability of analyses, simplifies the routine protocol and saves time. However, one step generally remains manual: dilution of the sample. Yet appliances dedicated to this use (called diluters) are commercially available. Schematically they are made of precision syringes activated by step by step motors. According to constructor specifications they achieve a high degree of precision (minimum RSD $<1 \%$ ) and accuracy (minimum RSD $<0.2 \%$ ).
The thing hindering expansion of diluters is the distrust of analysts toward appliances they are not used to using, and use of which have yet to be validated. We chose to validate the use of diluters in HPLC analyses by comparison with traditional, reliable and well-tried methods using a Pipette and Volumetric Flask, methods of which dispersion characteristics had been studied in depth [1]. An alternate method using a burette instead of pipettes was also evaluated. Data processing using statistical tools was described in detail to explain how information like precision or accuracy of the dilution method was obtained from experimental results.

* Correspondence and reprints.

Received October 27, 1998; revised January 19, 1999; accepted January 20, 1999. 


\section{Original articles}

Finally, the discussion established whether diluters could or could not advantageously replace traditional manual methods.

\section{Preamble}

The diluter used for the experiments was a Hamilton Microlab $530 \mathrm{~B}$ with two syringes. Preliminary qualification was undertaken by gravimetry using deionised water. Several samples of different volumes were weighed on a Mettler Toledo AG 245 analytical balance. Experimental data was in perfect agreement with manufacturer specifications: repeatability on levels reached was characterized by a RSD of less than $0.1 \%$ while average accuracy was around $0.2 \%$. Nevertheless, these good results were not sufficient to guarantee diluters perfectly suited for precision analyses. Gravimetry could in fact in no way account for the problems of memory effect and loss of product due to a possible mix between fluids. That is the reason why HPLC with UV detection had been chosen as a control in our experiments. First experiments tended to demonstrate, as expected, that the memory effect, and consequently the minimum ratio to be respected between the volumes of parent solution and diluent, dramatically depended on many parameters such as fluid viscosity, flow rate, temperature, etc. Since each situation was a special case and no general reliable rules could be established, we chose to bypass the difficulty. Instead of proceeding in a continuous manner, i.e. for each sample the parent solution and the diluent were sucked in and distributed in the same operation, we preferred a "fractionated" protocol. First the parent solution was sucked in and distributed for all the samples and then the diluent was distributed to all the samples. The fractionated protocol had the advantages of being quite insensitive to formerly quoted parameters, and of having a wider range of dilution ratios. The only precautions which had to be adopted were, on the one hand to fill the suction tubing using a volume of at least $500 \mu \mathrm{L}$ of parent solution, which acted as a "buffer" and suppressed fluid mixing of the solution of interest, and on the other hand to rinse abundantly the tubing between the parent solution and diluent.

\section{Experimental part}

\section{Product, HPLC method and injection sequence}

As explained before, the control was HPLC with UV detection. The product chosen to conduct the experiments was
Spiramycin [2]. This Rhône-Poulenc Rorer antibiotic may be considered a good example of the kind of products handled in the pharmaceutical industry.

Moreover, the HPLC method was fully validated and studied [3], and its dispersion characteristics were estimated rigorously through a collaborative study [4-6]. Briefly, this method used isocratic elution reversed phase chromatography. The column used was a Macherey Nagel Nucleosil $\mathrm{C}_{8}$ $120 \AA 3 \mu \mathrm{m} 200 \times 4.6 \mathrm{~mm}$. The mobile phase was a mixture of acetonitrile, and phosphate buffer $\mathrm{pH}=2.2$ (30:70, $\mathrm{v} / \mathrm{v})$ with $6.5 \mathrm{~g} / \mathrm{L}$ of sodium perchlorate. No specific supplier was requested; however, chemicals had to be HPLC grade and have successfully passed the conformity test (no $\mathrm{CN}^{-}$in acetonitrile). The flow rate was set at $0.8 \mathrm{~mL} / \mathrm{min}$. The injected volume was $20 \mu \mathrm{L}$. The detection wavelength was set at $232 \mathrm{~nm}$, and the column temperature had to be exactly $23{ }^{\circ} \mathrm{C}$. In these conditions, the analysis lasted about $35 \mathrm{~min}$.

To give greater confidence in the experimental results and in the conclusions, for each technique involving the skill of the operator (Pipette and Burette) the experiments were carried out in duplicate by two operators. Since automatic diluter performances were independent of the operator such a precaution was not necessary and a single operator was sufficient. Table I gives the detailed composition of HPLC devices used.

The injection sequence should enable various data elements to be given: the intrinsic repeatability of the chromatographic device, the additional variability introduced by the dilution technique (Pipette, burette or diluter), and its accuracy. Taking into account these considerations, the optimum sequence was a 6-level one: $25 \%, 50 \%, 75 \%, 100 \%$, $125 \%$ and $150 \%$ of the target value, corresponding to a concentration of $250 \mathrm{mg} / \mathrm{L}$ of Spiramycin, which gave an absorbance of about $0.4 \mathrm{AU}$ at the top of the main chromatographic peak. For each level, three independent preparations were prepared and each preparation was injected twice. If we call $X_{i}$ the $i^{\text {th }}$ preparation of the $X \%$ level and $B$ a blank, then the sequence can be written as follows:

$$
\begin{aligned}
& B / 25_{1} / 25_{2} / 25_{3} / 50_{1} / 50_{2} / 50_{3} / 75_{1} / 75_{2} / 75_{3} / 100_{1} / 100_{2} / 100_{3} / 125_{1} / \\
& 125_{2} / 125_{3} / 150_{1} / 150_{2} / 150_{3} / \mathrm{B} / 25_{1} / 25_{2} / 25_{3} / 50_{1} / 50_{2} / 50_{3} / 75_{1} / 75_{2} / \\
& 75_{3} / 100_{1} / 100_{2} / 100_{3} / 125_{1} / 125_{2} / 125_{3} / 150_{1} / 150_{2} / 150_{3}
\end{aligned}
$$

This sequence was used for each individual data set. A data set consisted of the results obtained by one operator

Table I. Components for both HPLC devices used.

\begin{tabular}{lccccc}
\hline Operator & Pump & Automatic injector & UV detector & Integrator & Solvent saver \\
\hline $\mathbf{1}$ & Varian & Waters 715 & Varian 2050 & Acquisition station & Ecosaver \\
& 9012 & Ultra Wisp & & Shimadzu Class VP & Jour Research \\
$\mathbf{2}$ & Varian & Basic & Spectromonitor & Merck D-2500 & 3000 \\
& 9012 & Marathon & 3200 LDC & & \\
\hline
\end{tabular}


using one dilution technique. Operator 1 tested in duplicate the three dilution techniques: pipette, burette and diluter while Operator 2 tested only once the two manual dilution techniques. Overall, 8 data sets were obtained.

\section{Experimental sample preparation}

All the samples used for a data set were obtained through dilution of a single parent solution. The parent solution was a solution of Spiramycin $1.25 \mathrm{~g} / \mathrm{L}$ in a mixture of water and acetonitrile $(70: 30 \mathrm{v} / \mathrm{v})$. It had a level of $500 \%$ compared to the target value. In fact, the accuracy of this value was not essential since the goal was not to obtain a calibration curve but to compare performances of the various dilution techniques.

\section{Pipettes and flasks}

This manual dilution technique was the reference one. It is in fact recognized by all the official organizations and has been used in most analytical laboratories for years. The protocol consisted in taking a given volume of the parent solution with a class A+ pipette, transferring it into a class $\mathrm{A}+$ volumetric flask, and adjusting to the desired volume with diluent. Diluent is a mixture of water and acetonitrile (70:30 $\mathrm{v} / \mathrm{v})$. Table II gives, for each level, the pipette and volumetric flask volumes used.

This technique was theoretically reliable, but the operator must be experienced and skilled. It was in any event somewhat time- and solvent-consuming.

\section{Burette and flasks}

This manual dilution technique was an adaptation of the former. Pipettes were replaced by a $10 \mathrm{~mL}$ class $\mathrm{A}+$ photophor burette. So it allowed time to be saved, and made the work of the operator easier. However its reliability was generally considered to be less good. Table III gives, for each level, the volumes distributed by the burette and the volumetric flask used.

\section{Diluter}

The Hamilton 530B was equipped with two syringes: $1000 \mu \mathrm{L}$ for the parent solution and $2500 \mu \mathrm{L}$ for the diluent. The fractionated protocol presented in section 2 was used. Samples were prepared directly in vials used by the automatic injector. Table IV gives the volumes of parent solution and of diluent used for each level.

No specific skill was required to run the dilution sequence.

\section{Compared characteristics of the dilution techniques}

In table $\mathrm{V}$ estimated characteristics of the three dilution techniques are given in terms of volumes and time. Values corresponded to the amount required for a complete data set. Times are merely rough estimates and depended heavily on operator speed.
Table II. Pipettes and flasks used.

\begin{tabular}{|c|c|c|}
\hline Level & Pipette volume $(\mathrm{mL})$ & Flask volume $(\mathrm{mL})$ \\
\hline $25 \%$ & 5 & 100 \\
\hline $50 \%$ & 5 & 50 \\
\hline $75 \%$ & 15 & 100 \\
\hline $100 \%$ & 10 & 50 \\
\hline $125 \%$ & 5 & 20 \\
\hline $150 \%$ & 15 & 50 \\
\hline
\end{tabular}

Table III. Volumes used for the burette dilution technique.

\begin{tabular}{lcc}
\hline Level & Distributed volume $(\mathrm{mL})$ & Flask volume $(\mathrm{mL})$ \\
\hline $25 \%$ & 2.5 & 50 \\
$50 \%$ & 2.5 & 25 \\
$75 \%$ & 7.5 & 50 \\
$100 \%$ & 5 & 25 \\
$125 \%$ & 5 & 20 \\
$150 \%$ & 7.5 & 25 \\
\hline
\end{tabular}

Table IV. Volumes used with the diluter.

\begin{tabular}{lcc}
\hline Level & Parent solution $(\mu L)$ & Diluent $(\mu L)$ \\
\hline $25 \%$ & 100 & 1900 \\
$50 \%$ & 200 & 1800 \\
$75 \%$ & 300 & 1700 \\
$100 \%$ & 400 & 1600 \\
$125 \%$ & 500 & 1500 \\
$150 \%$ & 600 & 1400 \\
\hline
\end{tabular}

\section{Data processing}

Quantification was achieved using peak area. Given the aim of the present work, a single peak area was sufficient to characterize the concentration level corresponding to each chromatogram. Indeed only values related to Spiramycin I, the main component in the Spiramycin, were taken into consideration.

Each data set was processed in the same way. The situation corresponded to a linear regression where an additional factor, i.e. the preparation, must be taken into account [7]. The theoretical expression for the response is shown in equation (1).

$$
y_{i j \alpha}=a+b x_{i}+c_{j(i)}+\varepsilon_{i j \alpha}
$$




\section{Original articles}

Table V. Compared characteristics of the dilution techniques.

\begin{tabular}{lccccc}
\hline Technique & Parent solution $(m L)$ & Diluent $(m L)$ & Time $($ hour $)$ & Skill level required & equipment \\
\hline Pipette & 165 & 945 & 2 & high & 3 pipettes and 18 flasks \\
Burette & 90 & 495 & 1.5 & high & 1 burette and 18 flasks \\
Diluter & $6.3+1.5$ ("buffer") & $29.7+75$ (rinse) & 0.5 & low & 1 diluter \\
\hline
\end{tabular}

where $y_{i j \alpha}$ : result of the $\alpha^{\text {th }}$ determination carried out on level $i$ with preparation $j(i)$,

$a$ : expected value of the response when $x=0$,

$c_{j(i)}$ : effect of the "preparation" factor at modality $j$, the level being $i$,

$\varepsilon_{i j \alpha}$ : effect of random error.

The ANOVA table corresponding to a data set is given in table VI.

To determine performances of the various dilution techniques, for each data set several elements of information must be extracted from the ANOVA. Firstly, repeatability variance of the HPLC device was estimated by the pure error mean square. To make comparison easier and to give meaningful values, reduced standard deviation was systematically used to characterize each source of variation. Equation (2) expresses estimate for repeatability reduced standard deviation.

$$
\hat{\sigma}_{r}=\frac{\sqrt{q_{\text {int } r a}}}{\hat{y}_{100 \%}} .
$$

Where $\hat{y}_{100 \%}$ is the value predicted by the model for the $100 \%$ level.

After this, the influence of the preparation factor was tested using an $\mathrm{F}$ test [8]. The $\alpha$ level was set at $5 \%$. If the preparation factor was found to be significantly influential it was possible to give an estimate of its reduced standard deviation, see equation (3).

$$
\hat{\sigma}_{\text {prep }}=\frac{\sqrt{\left(q_{\text {prep }}-q_{\text {int ra }}\right) / 2}}{\hat{y}_{100 \%}} .
$$

Usually, in linear regression, Anova is used to test the validity of the model thanks to the lack of fit test [8-9]. Here, the validity of the model depends on the accuracy of the dilution techniques and was checked by means of the modified lack of fit test [7]. If the lack of fit test did not reject the appropriateness of the linear model, the sensitivity of the analysis method was constant and so the dilution method was accurate. If not, the accuracy of the method could be questioned. In addition, a visual examination of residuals was used to detect suspect behavior.
Table VI. ANOVA table for a data set.

\begin{tabular}{lccc}
\hline $\begin{array}{l}\text { Source of } \\
\text { variation }\end{array}$ & $\begin{array}{c}\text { Sum of } \\
\text { squares }\end{array}$ & $\begin{array}{c}\text { Degrees of } \\
\text { freedom }\end{array}$ & $\begin{array}{c}\text { Mean } \\
\text { square }\end{array}$ \\
\hline Regression & $Q_{\mathrm{R}}=b^{2} \sum_{i}\left(x_{i}-\bar{x}\right)^{2}$ & 1 & $q_{\mathrm{R}}=b^{2} \sum_{i}\left(x_{i}-\bar{x}\right)^{2}$ \\
Lack of fit & $Q_{\mathrm{L}}=\sum_{i}\left(\bar{y}_{i}-\hat{y}_{i}\right)^{2}$ & $6-2=4$ & $q_{\mathrm{L}}=Q_{\mathrm{L} / 4}$ \\
Preparation & $Q_{\text {prep }}=\sum_{i j}\left(\bar{y}_{i j}-\bar{y}_{i}\right)^{2}$ & $6(3-1)=12$ & $q_{\text {prep }}=Q_{\mathrm{prep}} / 12$ \\
Pure error & $Q_{\text {int ra }}=\sum_{i j \alpha}\left(y_{i j \alpha}-\bar{y}_{i j}\right)^{2}$ & $36-6 * 3=18$ & $q_{\text {int ra }}=Q_{\text {int ra }} / 18$ \\
\hline
\end{tabular}

Table VII. Experimental results

\begin{tabular}{lccccc}
\hline Operator & $\begin{array}{c}\text { Dilution } \\
\text { method }\end{array}$ & $\begin{array}{c}\hat{\sigma}_{r} \\
\text { Influence of } \\
\text { preparation } \\
(\alpha=5 \%)\end{array}$ & $\hat{\sigma}_{\text {prep }}$ & $\begin{array}{c}\text { Sensitivity } \\
\text { Constancy }\end{array}$ \\
\hline$\# 1$ (run 1) & Pipettes & $0.26 \%$ & yes & $0.25 \%$ & yes $(\alpha=1 \%)$ \\
$\# 1$ (run 1) & Burette & $0.28 \%$ & yes & $0.48 \%$ & yes $(\alpha=5 \%)$ \\
$\# 1$ (run 1) & Diluter & $0.23 \%$ & yes & $0.23 \%$ & yes $(\alpha=5 \%)$ \\
$\# 1$ (run 2) & Pipettes & $0.26 \%$ & yes & $0.53 \%$ & yes $(\alpha=5 \%)$ \\
$\# 1$ (run 2) & Burette & $0.21 \%$ & yes & $0.38 \%$ & yes $(\alpha=5 \%)$ \\
$\# 1$ (run 2) & Diluter & $0.36 \%$ & yes & $0.34 \%$ & yes $(\alpha=5 \%)$ \\
$\# 2$ & Pipettes & $0.44 \%$ & yes & $0.52 \%$ & yes $(\alpha=1 \%)$ \\
$\# 2$ & Burette & $0.50 \%$ & no & $<0.41 \%{ }^{*}$ & no \\
\hline
\end{tabular}

*Value obtained with $\alpha=5 \%$.

Experimental design and the source of the various elements of information are illustrated in figure 1 .

\section{Results and discussion}

All the required calculations were achieved with the help of the application $\mathrm{JMP}^{\circledR}$ for Windows [10]. Experimental results obtained for the eight data sets are shown in table VII.

The repeatability of the two HPLC devices differed slightly, but in both cases estimates obtained were quite 


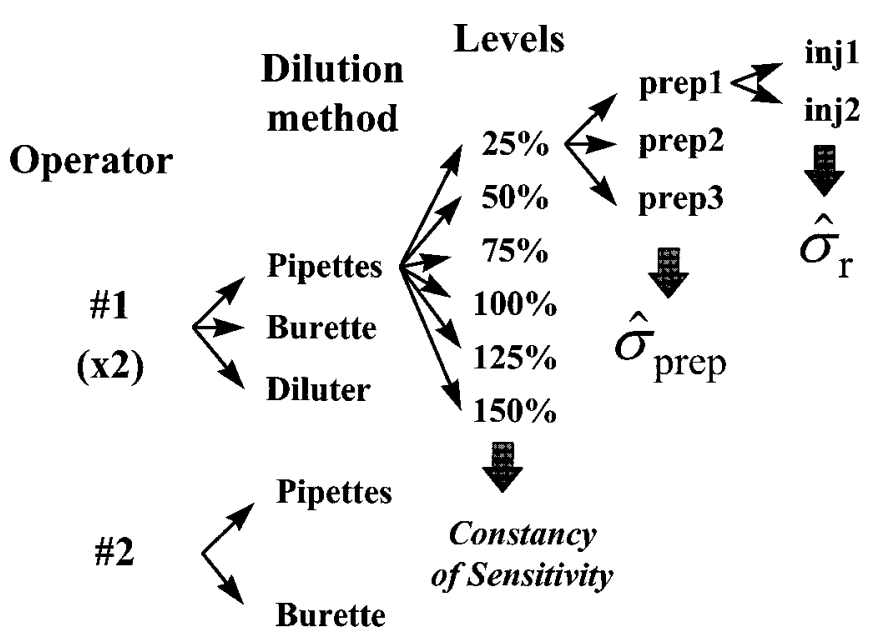

Fig. 1. Experimental design scheme.

consistent with the value $0.39 \%$ obtained during the collaborative study [5].

For all data sets the preparation factor was found to be significantly influential. Only operator \#2 on the experiment with a burette did not find significant influence for the preparation. However, statistical reasoning established that for this data set the preparation reduced standard deviation could under no circumstances exceed $0.41 \%$. The dispersion introduced by the Pipette dilution technique ranged from $0.25 \%$ to $0.53 \%$, with an average value of $0.45 \%$. No significant differences between operators was detected since even for a single operator the value could vary by a factor of 2. The dispersion introduced by the Burette dilution technique ranged from $0.38 \%$ to $0.48 \%$, with an average value of $0.43 \%$. In respect of the dispersion introduced, the burette dilution technique seemed as good as, or better than, the reference dilution technique with Pipettes. The dispersion introduced by diluter dilution technique ranged from $0.23 \%$ to $0.34 \%$, with an average value of $0.29 \%$. This is the dilution technique which experimentally exhibited the smaller dispersion, and in addition differences between data sets are less important than for other techniques. It meant a more robust dilution technique which was less sensitive to the skill and stress of the operator. Nevertheless, at this stage, differences observed between techniques were too small to draw decisive conclusions.

The constancy of sensitivity, i.e. the accuracy of the dilution technique, was always satisfactory, except for the burette. The data set of operator \#2 showed problems with regard to accuracy of the level value, which was confirmed by a visual examination of the regression residuals. As a consequence, this technique must be considered to be insufficiently accurate, and was not trustworthy enough to be used in a definitive validation procedure.

The diluter dilution technique appeared accurate and more precise than the reference technique with pipettes and volu- metric flasks. So its use could be de facto considered validated. The impact was huge since although the benefit was somewhat reduced in terms of the quality of the results, the diluter allowed considerable saving in relation to the quantity of product (20 times less) and the volume of solvent used as diluent (4 times less). The time saved was also substantial since experimental preparation lasted only $30 \mathrm{~min}$, compared to 2 hours with pipettes. Another argument in favor of the diluter was the lower level of stress imposed on the operator. Consequently, the likelihood of making a mistake was smaller with a diluter.

Some people could still be reluctant to use diluters, arguing that they could be disturbed, and produce biased values. Indeed, to prevent such discrepancies, like any other instrument, diluters need to be checked, calibrated and maintained regularly [11]. The time spent in checking or maintenance would in any event easily be recovered.

\section{Conclusion}

The diluter dilution technique was easily validated by experimental comparison with the reference dilution technique based on pipettes and volumetric flasks. Performances obtained with the diluter were at least as good as, or better than, those obtained with the reference technique. This was true not only for the dispersion characteristics but also for the accuracy of the level targeted. The impact of this validation is less in the improvement of the overall performances than in terms of convenience of use and in the overall reduction of the analysis cost. In spite of the results obtained here it must be borne in mind that use of a diluter requires that some rules are followed, especially concerning the protocol. An inappropriate protocol can lead to biased results and counteract all the advantages of the diluter. In addition, it is essential to ensure that the diluter works well, and this point can only be ensured with periodical verification. In any event, it has been proven that diluters can advantageously replace manual dilution techniques with pipettes and flasks, and it would be a pity not to use this appliance to improve the quality and reduce the cost of many analyses

\section{Acknowledgment}

We would like to thank particularly Rhône-Poulenc Rorer (Centre de Recherche de Vitry Alfortville - France), which supported us in our research and experiments.

\section{References}

1. Hayashi, Y.; Matsuda, R Anal. Sci. 1994, 10, 881-888.

2. French Pharmacopoeia, $10^{\text {th }}$ ed., ADRAPHARM, Maisonneuve S.A., Paris, 1989.

3. Vial, J.; Jardy, A. Analusis 1998, 26, 15-24.

4. Jardy, A.; Vial, J.; Ménier, I. Analusis 1997, 25, 106-111.

5. Vial, J.; Ménier, I.; Jardy, A.; Anger, P.; Brun, A.; Burbaud, L. J. Chromatogr. B 1998, 708, 131-143. 


\section{Original articles}

6. Vial, J.; Jardy, A.; Anger, P.; Brun, A.; Menet, J. M. J. Chromatogr. A 1998, 815, 173-182.

7. Vial, J.; Jardy, A. submitted for publication.

8. Commissariat à l'Énergie Atomique, Statistique appliquée à l'exploitation des mesures, I et II, Masson, Paris, France, 1978.
9. Massart, D. L.; Vandeginste, B. G. M.; Deming, S. N.; Michotte, Y.; Kaufman, L. In Chemometrics: a textbook, Elsevier, Amsterdam, 1988; p 81.

10. JMP Statistic and Graphics Guide and User's guide (Version 3.1), SAS Institute Inc., Cary, NC, USA, 1995.

11. Meunier, F. Spectr. Anal. 1998. 